\title{
A Fixed Gap APPLE II Undulator for SLS
}

\author{
T. Schmidt, A. Imhof, G. Ingold, B. Jakob and C. Vollenweider \\ Paul Scherrer Institut, Swiss Light Source, 5232 Villigen PSI, Switzerland
}

\begin{abstract}
To vary the polarization vector of an APPLE II undulator continuously from $0-180^{\circ}$, all four magnet arrays need to be movable. Following the adjustable-phase undulator approach by R. Carr, a $3.4 \mathrm{~m}$ long fixed gap undulator for SLS with a gap of $11.6 \mathrm{~mm}$ has been constructed. It will be installed in fall 2006. The gap drive is replaced by a pair-wise shift of the magnet arrays to change the energy, while the polarization is changed by shifts of diagonal arrays. The high injection efficiency and standard operation top-up mode at the SLS allows this simplified undulator design. The design as well as the operational aspects will be discussed.
\end{abstract}

Keywords: Undulator, APPLE II

PACS: $07,85 . \mathrm{Qe}, 41.85 . \mathrm{Lc}$

\section{INTRODUCTION}

As the last ID to be installed at the $2.4 \mathrm{GeV}$ Swiss Light Source (SLS), the UE44 fixed gap APPLE II undulator will provide light within $350-2000 \mathrm{eV}$ for the ADRESS beamline, covering the $\mathrm{L}$ edges of the transition metals and the rare earth $\mathrm{M}$ edges. The beamline is dedicated to resonant inelastic $\mathrm{x}$-ray scattering (RIXS) for which high flux and high resolving power are needed. All polarization modes are required: linear horizontal and vertical, variable angle between $0-180^{\circ}$ and circular. The flux has been optimized at $1 \mathrm{keV}$ while guaranteeing overlap between the first and third harmonic in all operation modes. The beamline will have a micro-focus of $10 \mu \mathrm{m}$, so the possibility to rotate the polarization of the light instead of the sample is of high importance. The SLS will be fully equipped in fall

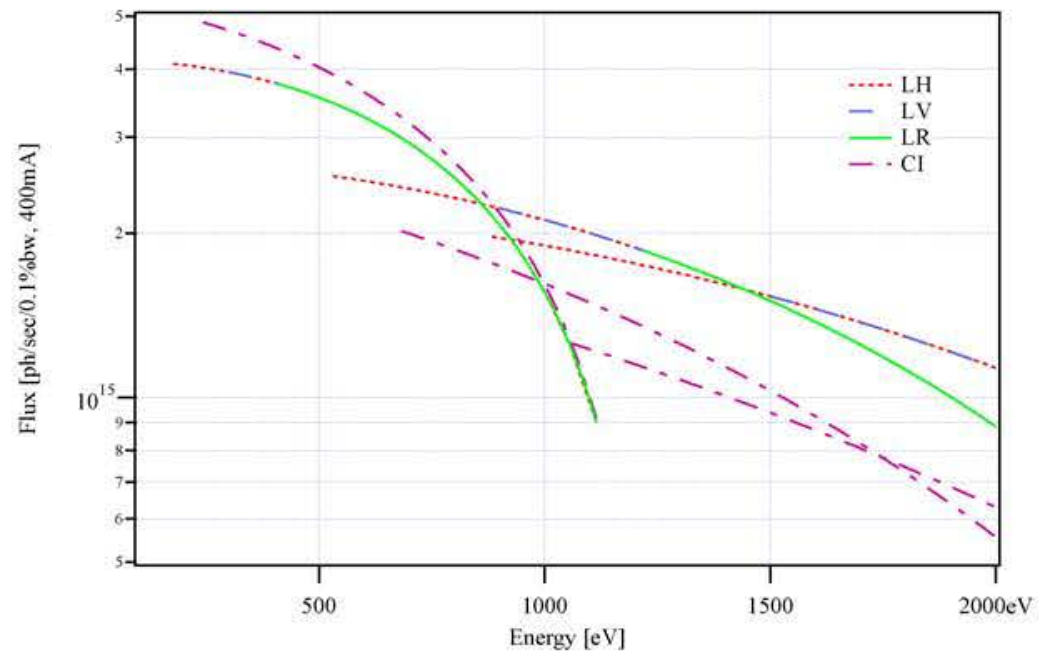

FIGURE 1. Spectra of UE44 undulator for the ADRESS beamline for all operation modes. For all linear polarizations (lin. horizontal LH, lin. vertical LV and lin. variable LR), the flux curves are on top of each other, but the minimum energies vary. In circular mode, the higher harmonics provide elliptical light.

2006, within five years after the inauguration in August 2001, with altogether 12 insertion devices: 4 small gap, small period in-vacuum undulators [1], 4.APPLE II undulators, 2 elliptical quasi-periodic electromagnetic undulators and 2 wigglers, providing light for 9 beamlines.

CP879, Synchrotron Radiation Instrumentation: Ninth International Conference, edited by Jae-Young Choi and Seungyu Rah

(C) 2007 American Institute of Physics 978-0-7354-0373-4/07/\$23.00 


\section{THE UE44 DESIGN}

The undulator has a period length of $44 \mathrm{~mm}$ and with 75 periods the overall length is about $3.4 \mathrm{~m}$. NdFeB magnets with a remanence of $B_{r}=1.24 \mathrm{~T}$ result in flux densities of $B_{y}=0.85 \mathrm{~T}$ and $B_{x}=0.61 \mathrm{~T}$, or K-values of 3.5 and 2.5 respectively. To achieve this, a rather small gap of $11.6 \mathrm{~mm}$ is needed. The vacuum chamber has a vacuum stay clear aperture of $8 \mathrm{~mm}$ with $1 \mathrm{~mm}$ wall thickness. It is made of extruded aluminum without antechamber, machined at PSI and NEG coated at ESRF.

\begin{tabular}{lrr}
\multicolumn{3}{c}{ TABLE 1. Parameter of the UE44. } \\
\hline typc & APPLE II, fixed gap \\
period length & $44 \mathrm{~mm}$ & 75 \\
periods / length & NdFel3 $B_{r}=1.24 \mathrm{~T}$ & $(30 \times 30 \times 10.7) \mathrm{mm}^{3}$ \\
magnets & vacuum: $8 \mathrm{~mm}$ & magnetic: $11.6 \mathrm{~mm}$ \\
gap & $B_{y}=0.85 \mathrm{~T}$ & $B_{x}=0.61 \mathrm{~T}$ \\
flux density & $K_{y}=3.5$ & $K_{x}=2.5$ \\
$\mathrm{~K}$ - value & $\mathrm{LH}, \mathrm{LV}, 0-180^{\circ}, \mathrm{circ}$ & \\
operation modes & $2 \cdot 10^{15} \mathrm{ph} / \mathrm{sec} / 0.1 \% \mathrm{bw}$ & \\
flux at $1 \mathrm{keV}$ & &
\end{tabular}

The demand to rotate the linear polarization vector the by $180^{\circ}$ requires four shiftable magnet arrays [5]. This implies a new design of the shift-section of the undulator and increases the number of motors to six: four shift axes and two gap axes ${ }^{1}$. Having four shift axes, however, already allows a full control of both energy and polarization of the undulator, as has been pointed out by R. Carr and S. Lidia [6] [7]. For the UE44 this design concept has been adapted, not by replacing the gap drive by an additional shift drive, but rather by using the shift drives needed for polarization control also for the energy control. Operating the undulator at a fixed gap with a constant ratio of the vertical and horizontal fields seems to be favorable and it allows for a simplified design of the support structure.

Of course, the fixed gap design has the potential risk of radiation damage to the magnets, because it cannot be opened for injection and is operated always at the smallest gap. However, the SLS is running $100 \%$ in top-up mode, has a very efficient injection (i.e. due to the low emittance booster: $7 \mathrm{~nm}$ rad compared to $5 \mathrm{~nm}$ rad of the storage ring), and although the gap is rather small, the vacuum chamber is not the limiting vertical aperture. The magnetic field cannot be switched off, but the lateral components $B_{y}$ and $B_{x}$ will be transformed into the longitudinal component $B_{x}$. However, the longitudinal fields have already been introduced with the asymmetric shift-mode for the linear variation of the polarization vector. The focusing effects, however, scale with period length and are harmless to the SLS beam dynamics.

\section{MECHANICS}

The design of the support structure of the undulator is based on a simple but stiff welding construction with a minimum number of accurate surfaces. The production in one undivided unit was incompatible with the desired overall dimensions. The resulting vertical separation in the beam plane caused more machining work, but the accuracy achieved is excellent: without load, the bearing surfaces are measured to be within $\pm 25 \mu \mathrm{m}$, with a parallelism of $50 \mu \mathrm{m}$. This separation allowed in return a convenient assembly of the I-beam and the magnets. The whole support structure is placed in the ring tunnel on three lateral rails which allow to move the undulator precisely over its vacuum chamber. For the shift system with 4 individual arrays, the number of parts should be minimized. There is only one common lateral reference for both magnet arrays on one I-beam, the central double $\mathrm{V}$ guide rail, positioned to one flange of the groove. The double $\mathrm{M}$ shift arrays are machined from stainless steel, but have integrated hardened guiding surfaces, which have been electron beam welded at PSI. The final grinding has been carried out in one clamping with the stop faces for the modules carrying the magnets. The shift arrays have a length of $1.7 \mathrm{~m}$. Two of them are connected by the shift lever, which brings in the force from the drive system. The magnets are clamped in aluminum keepers, which are grouped to modules. Both modules and keeper are positioned in the longitudinal direction by precision pins.

\footnotetext{
I at the SIS, the [I)s have two gap motors to correct for small taper errors. I lowever, the [I)s have no taper option, which widens the harmonics. IDs and monochromators are driven by the same control system which enables a good overlap, offering the higher flux or brilliance.
} 

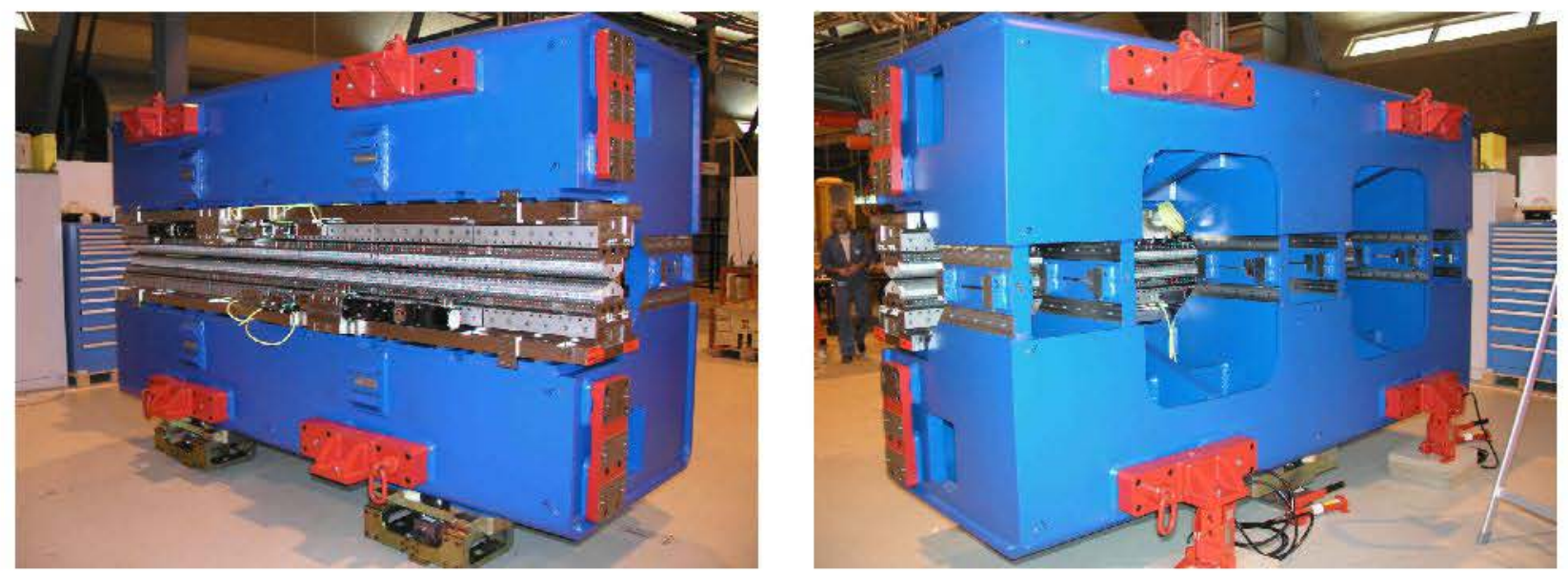

FIGURE 2. Front and back side of the fixed gap l:F44. The support structure is a heavy, but simple and stif' welding construction.

The magnets have slightly tilted shoulders, which produce in addition to the main force component also a minor one, which should help to fix the magnets in the keeper. The keeper is fixed by two vertical screws. One screw fixes the magnets on the modules, the second goes through the module and fixes the keeper and modules onto the shilt array.
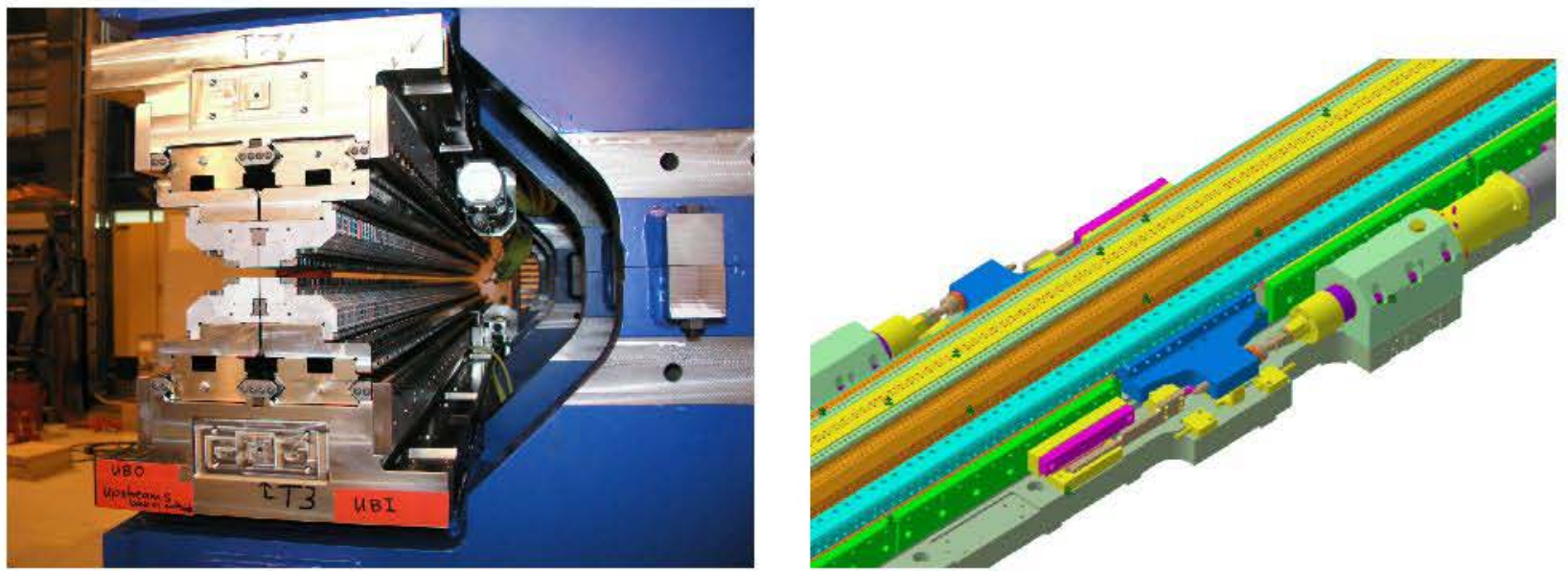

HIGURE 3. I.eft: central part of the I!H44 shows I beam with modules, keeper and magnets. The center double- $V$ guidance is the common reference for both arrays on the stainless stecl I-bcam. Right: the drive chain with shift lever and linear cncoder system.

The drive system consists of de servo motors, a pneumatic brake, a 1:35 gear box, a spindel and a shift lever, which connects the two shift arrays in one row. The correct positioning is done in closed loop with the direct shift position reading by a Heidenhain linear encoder. The measuring system is attached on the l-beam and at the lever arm just below the guided shift arrays. Measurements of the stability of the drive system gave under the longitudinal force from one l-beam of $14 \mathrm{kN}$ an elasticity of $130, \mu \mathrm{m}$. As the system adjusts to the linear encoder signal, this is not harmful. The relevant movement of the measurement lever to the magnet array is only $2, u \mathrm{~m}$, which corresponds to an energy shift of $\Delta E / E=1 \cdot 10^{-4}$. The photo shows the fully assembled undulator. The details of the magnet part and shift lever with measurement system are presented as $3 \mathrm{D}$ construction drawings for a more detailed view.

The mechanical components are of excellent quality. Measurements with a Leica laser tracker showed parallelism of the relevant planes within a band of $50 \mu \mathrm{m}$. Finite Element Calculations of the support structure predict maximum $30 \mu \mathrm{m}$ variation, when the vertical forces change from $\pm 25 \mathrm{kN}$. The measured variation is $24 \mu \mathrm{m}$, asymmetrically distributed to $19 \mu \mathrm{m}$ from maximum vertical fields to the zero vertical fields and additional $5 \mu \mathrm{m}$ at repelling vertical forces. For being secure, two clamps on the gap site of the C-shaped structure are foreseen which could improve the stability. 


\section{MAGNETICS}

The magnets are grouped and measured magnetically on modules carrying 3 or 5 magnets, which allows zero integral measurements. First the individual magnets have been sorted based on on-axis field data calculated with RADIA from the suppliers magnet data $B_{r}, \alpha_{x}$ and $\alpha_{y}$. Measurements of the modules with moving wire and on-axis Hall probes are then the basis for the distribution of the modules on the shift arrays. Sortings for both, magnets to modules and modules to shift arrays have been carried out using a simulated annealing algorithm. The modules have been sorted on the phase error in $\mathrm{x}$ - and $\mathrm{y}$-direction (optionally including on and off axis field integrals, not used here).

\section{OPERATION}

To set the correct phase and energy shifts $(\phi, \rho)$ for the input parameter energy and polarization $(E, \tan \alpha)$ in circular mode, the fixed gap undulator can be analytically described. Once the correct phase shift has been set depending on the harmonic, the energy shift as function of the photon energy can be calculated[2]:

$$
\begin{aligned}
\phi & =2 \arctan R_{h} \frac{K_{z 0}}{K_{x 0}}, \quad R_{h}=1(0.8,0.6) \\
\rho & =2 \arccos \sqrt{2\left(\frac{C}{E}-1\right) \frac{1}{K_{z 0}^{2} \cos ^{2} \frac{\phi}{2}+K_{x 0}^{2} \sin ^{2} \frac{\phi}{2}}}
\end{aligned}
$$

For the linear mode, $\rho$ has to be implemented in the equation for the photon energy $E$, which has to be solved numerically to the phase shift $\phi$. The second step is to calculate the energy shift $\rho$.

$$
\begin{aligned}
E & =\frac{C}{1+0.5\left(K_{z 0}^{2} \cos ^{2} \frac{\phi}{2} \cos ^{2} \frac{\phi+\rho}{2}+K_{x 0}^{2} \sin ^{2} \frac{\phi}{2} \sin ^{2} \frac{\phi+\rho}{2}\right)} \\
\rho & =2 * \arctan \left(\frac{K_{z 0}}{K_{x 0}} \cot \frac{\phi}{2} \cot \alpha\right)-\phi
\end{aligned}
$$
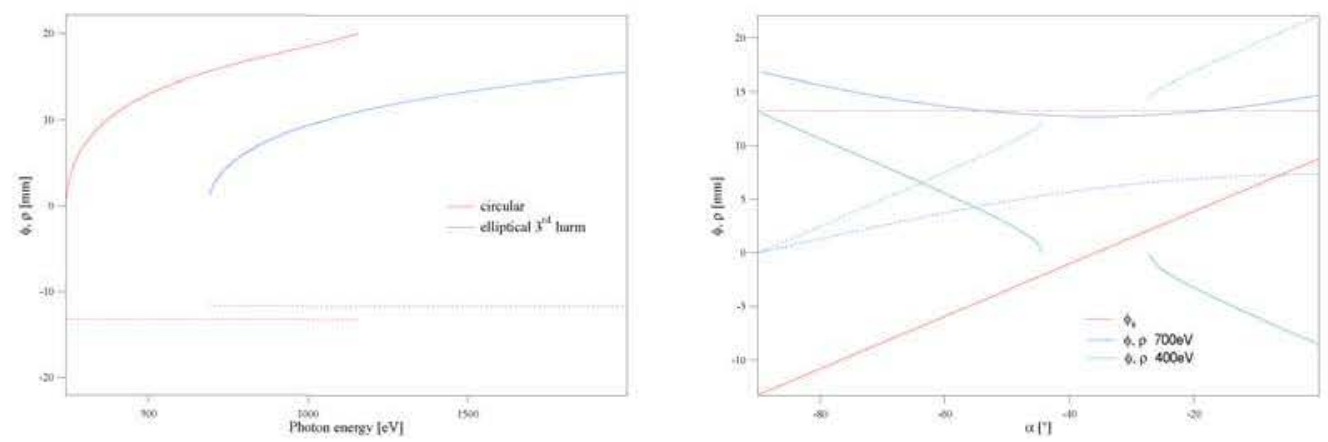

FIGURE 4. Phase $\phi$ (dotted lines) and energy shift $\rho$ (solid lines), left in circular mode, right in linear polarization mode. In circular mode $\phi$ is fixed and $\rho$ is a function of energy. The right diagram shows examples for different energies: At the symmetry phase condition $\phi_{s}, \rho$ varies linearly with the angle $\alpha$ [2]. For lower energies, there is a gap in reachable polarization angles. For a rotation over the entire range $180^{\circ}$, the other diagonal pair of magnet rows has to be shifted.

\section{REFERENCES}

1. G. Ingold et al., Performance of Small-Gap Undulators at the SLS Intermediate Energy Storage Ring, these proceedings.

2. T. Schmidt, D. Zimoch, About Apple Operation, these proceedings.

3. S. Sasaki et al., Design of a new type of planar undulator for generating variably polarized radiation, Nucl. Instr. and Meth. A 331, 763-767 (1993).

5. J. Bahrdt et al., Undulators for the Bessy soft-x-ray FEL, Proceedings of the 2004 FEL Conference, Trieste, $610-613$ (2004).

6. R. Carr, Adjustable phase insertion devices as X-ray source, Nucl. Instr. and Meth. A306, 391 (1991).

7. S. Lidia, R. Carr, An elliptically-polarizing undulator with phase adjustable energy and polarization, Nucl. Instr. and Meth. A 347, 77-82 (1994). 